\title{
Genomic analysis of perinatal sucking reflex in German Brown Swiss calves
}

\author{
C. Dreher, ${ }^{1}$ R. Wellmann, ${ }^{1}$ P. Stratz, ${ }^{1}$ M. Schmid, ${ }^{1}$ S. Preuß,${ }^{1}$ H. Hamann, ${ }^{2}$ and J. Bennewitz ${ }^{1 *}$ \\ ${ }^{1}$ Institute of Animal Science, University of Hohenheim, 70599 Stuttgart, Germany \\ ${ }^{2}$ State Office for Spatial Information and Land Development Baden-Wuerttemberg (LGL), 70806 Kornwestheim, Germany
}

\begin{abstract}
A healthy sucking reflex is essential for newborn calves to ensure sufficient colostrum intake in the first few hours postpartum. In recent decades, European Brown Swiss breeders have repeatedly reported that some calves lack the ability to consume colostrum directly after birth due to an absent sucking reflex. In this study, we collected the phenotypes of more than 5,500 German Brown Swiss calves and performed variance component estimation with sire threshold models using Markov chain Monte Carlo algorithms. The 50K (777K) genotypes of nearly 2,000 (200) calves were collected, and an imputation was performed for all $50 \mathrm{~K}$ genotypes up to $777 \mathrm{~K}$. Genome-wide association studies (GWAS) for the trait sucking reflex were conducted for all 777K genotypes. Depending on the trait coding, a low heritability was estimated to range from 0.08 to 0.11. The GWAS results identified 34 trait-associated SNP on 6 different chromosomes. Post-GWAS analyses showed significant overrepresentation of Gene Ontologies for central nervous development and several regulative processes. Functional annotation clustering and pathway analysis revealed relations to lipid metabolism, immune and endocrine systems, and signal transduction. The results of this study suggest that breeding for an improved sucking reflex is possible but requires large data sets for the estimation of reliable breeding values (either large progeny testing groups or a large reference genome in a genomic selection program).
\end{abstract}

Key words: Brown Swiss calf, perinatal sucking reflex, genetic parameter, genome-wide association study

\section{INTRODUCTION}

It is well known that a sufficient colostrum intake in the first few hours after birth is of fundamental importance for the passive immunization of newborn calves. Several studies have discussed the consequences

Received February 13, 2019.

Accepted March 12, 2019.

*Corresponding author: j.bennewitz@uni-hohenheim.de of a failed passive transfer of immunity and the effects of environmental factors on colostrum intake (e.g., Weaver et al., 2000; Beam et al., 2009; Vasseur et al., 2009; Windeyer et al., 2014; Homerosky et al., 2017). Insufficient colostrum intake not only leads to poor growth and improper development, but it is also considered one of the main reasons for postnatal mortality (Hadorn et al., 1997; Weaver et al., 2000; Windeyer et al., 2014; Gomez and Chamorro, 2017). Therefore, insufficient colostrum intake is an animal welfare problem and is of high economic interest. A well-developed sucking reflex is the basic prerequisite for voluntary and adequate colostrum intake. However, Brown Swiss breeders in South Germany have repeatedly reported that some newborn Brown Swiss calves do not develop a sufficient sucking reflex within the first few hours postpartum (Just et al., 2018). This pattern has also been previously reported for the Italian Brown Swiss population (Maltecca et al., 2007). For calves with an insufficient sucking reflex, the farmers have to either invest considerable effort, working time, and patience to supply colostrum to the calf by hand or use esophageal tubes. The improper use of esophageal tubes poses a considerable risk for future lung diseases, which is why farmers often hesitate or even refuse to apply this tool.

Vasseur et al. (2009) examined several environmental factors affecting the absorption of colostrum by calves. They found that colostrum intake was best predicted by a combination of birth weight and the calf's degree of vigor. They did not find any effects of sex, calving ease, or time the first colostrum was offered. Furthermore, the trace element selenium has an effect on the sucking reflex in newborn livestock (Bostedt and Schramel, 1990; Reed et al., 2007; Hefnawy and Tórtora-Pérez, 2010; Konvičná and Kováč, 2015). It is common practice to supplement nonsucking calves with selenium and vitamin E.

Little is known about the genetic background of the trait sucking reflex in newborn calves. Maltecca et al. (2007) estimated the genetic parameters for perinatal sucking behavior in Brown Swiss calves and reported small to moderate heritabilities, which were based on the model used and the trait definition. These findings 
clearly indicate that a calf's genetic background has an effect on the expression of the sucking reflex. Furthermore, the implementation of a breeding strategy to improve the sucking reflex in newborn Brown Swiss calves could be possible. A recent survey revealed that German Brown Swiss breeders assigned a relatively high economic weight to perinatal sucking behavior (Just et al., 2018), indicating that the inclusion of this trait in the selection index is desired. Genomic selection was introduced in German Brown Swiss cattle breeding several years ago. The version of the Illumina BovineSNP50 v2 BeadChip used for this purpose contained a number of breed-specific SNP that were associated with juvenile mortality (Schwarzenbacher et al., 2016). If the genetic variation of sucking behavior can be tagged by only a few SNP, these might be included in the chip in a similar vein, which would simplify breeding for this trait. On the other hand, if no such SNP can be identified, the trait has to be treated as a typical quantitative trait, and continuous data collection is needed.

The aim of the present study was to estimate the genetic parameters for the trait perinatal sucking reflex in German Brown Swiss cattle and conduct GWAS to clarify the genetic architecture of the trait. Furthermore, it was of special interest to identify trait-associated SNP that might be important for genome-based breeding efforts.

\section{MATERIALS AND METHODS}

\section{Phenotypic and Pedigree Data}

The collection of phenotype data and tissue samples was performed between June 2015 and July 2016 on 224 farms in southwest Germany. The participation of the farm managers in the project was voluntary. The participating farms were preselected through the breeding organization to ensure a suitable data structure. Farm managers and employees were trained in recording the phenotypes of interest to ensure a high level of standardization.

Phenotypes were recorded for a total of 9,307 calves. The following characteristics were acquired for every newborn calf on the participating farms during the data collection period. The sucking reflex was measured by putting one finger into the calf's mouth and capturing the strength of the reflex. This was recorded on an ordinal scale ranging from 1 to 4 , with 1 representing calves with no sucking reflex at all and 2 representing calves that showed at least a weak sucking reflex. Calves with a normal sucking reflex were given a 3 , and a strong sucking reflex was represented by 4 . The summary statistics of the edited data set for the trait sucking reflex are shown in Table 1. In addition, the size and vigor of
Table 1. Description of the trait categories and number of observations (n) within the categories for the sucking reflex

\begin{tabular}{lccccc}
\hline & \multicolumn{4}{c}{ Category and number of observations } & \\
\cline { 2 - 5 } Trait category & 1 & 2 & 3 & 4 & Sum \\
\hline Sucking reflex & None & Weak & Normal & Strong & \\
$\mathrm{n}$ & 530 & 801 & 3,462 & 748 & 5,541 \\
\hline
\end{tabular}

each calf were assessed after calving, with 1 representing light and small calves, 2 representing calves of medium size and heavy, and large calves represented by a 3 . For vigor, weak calves were denoted by 1 and vital calves by 2 . The farmers documented the twin status, calving ease, duration of the first colostrum offering, first successful colostrum intake, abnormalities, malfunctions, and diseases or infections. All crossbred calves, calves with abnormalities or malfunctions, or calves that did not survive the first $48 \mathrm{~h}$ postpartum were excluded from further analysis. This was done because a clear assessment of the sucking reflex was not possible for these calves. Gestation lengths of the dams were provided by the State Office for Spatial Information and Land Development Baden-Wuerttemberg (Germany). Preliminary analysis revealed a strong farm effect on the sucking reflex. Therefore, to be able to estimate the farm effect precisely, herds with fewer than 15 observed calves were discarded from further analysis. This resulted in 5,541 calves, descending from 178 sires and 108 herds that were used for pedigree-based variance component estimation. The pedigree of the sires was traced back 5 generations.

The effects of explanatory variables were analyzed using the Wald test implemented in ASReml-R version 3.0 (Butler, 2009). The following variables were simultaneously tested for significance for the trait sucking reflex: herd, twin status, size and vigor of the calf, sex, season (2 seasons), calving ease, and gestation length. Variables with significant influence on the trait were included as fixed effects in further analyses.

\section{Genotype Data}

Tissue samples of more than 5,000 calves were collected from ear tags by staff members of the breeding organization. For genomic analysis, a subset of 3,072 calves was genotyped with the Illumina BovineSNP50 v2 BeadChip (Illumina Inc., San Diego, CA). The SNP filtering was performed using Illumina GenomeStudio V2011.1. Individuals with more than 10\% missing marker genotypes were removed. Only autosomal SNP with call frequency $\geq 0.99$ and cluster separation $\geq 0.4$ remained in the data set. Further SNP filtering was conducted using PLINK v1.07 (Purcell et al., 2007), 
Table 2. Trait coding (TC) of the trait sucking reflex, mean, and SD of the posterior distribution of the heritability with corresponding lower (l-HPD) and upper bounds of the highest posterior density (u-HPD)

\begin{tabular}{llcccc}
\hline $\begin{array}{l}\text { Trait } \\
\text { coding }\end{array}$ & Categories & $\mathrm{h}^{2}$ & $\mathrm{SD}$ & $\mathrm{l}-\mathrm{HPD}$ & $\mathrm{u}-\mathrm{HPD}$ \\
\hline TC1 & 1 (no), 2 (weak), 3 (normal), 4 (strong) & 0.110 & 0.056 & 0.027 & 0.22 \\
TC2 & 1 (no), 0 (weak, normal, strong) & 0.084 & 0.047 & 0.013 & 0.19 \\
TC3 & 1 (no, weak), 0 (normal, strong) & 0.095 & 0.057 & 0.019 & 0.19 \\
\hline
\end{tabular}

with minor allele frequency $<0.03$ and a significant deviation from the Hardy-Weinberg equilibrium $(P$ $<0.001$ ), leaving a total of 36,567 SNP in the data set. The phasing and imputation of sporadic missing genotypes was done using Beagle software version 3.3.2 (Browning and Browning, 2009). A total of 192 calves were additionally genotyped with the Illumina BovineHD BeadChip. For this purpose, preference was given to pairs of half sibs that were raised on the same farm but showed divergent sucking reflex. The SNP filtering was conducted as described for the $50 \mathrm{~K}$ genotypes. To obtain higher density marker information for all genotyped individuals, 50K genotypes were imputed using Minimac3 (Howie et al., 2012). The imputation accuracy was assessed by 5 -fold cross-validation and was, on average, 0.95 (not shown elsewhere). After quality control (filtering criteria, see above), high-density genotypes for 1,871 calves with known phenotypes and 525,949 SNP remained in the data set.

\section{Variance Component Estimation}

Three different trait coding (TC) approaches were used. The first trait coding (TC1) approach used the 4-class ordinal scale that was recorded by the farmers (Table 1). The second (TC2) combined classes 2, 3, and 4 into 1 class; and trait coding 3 (TC3) combined classes 1 and 2 and classes 3 and 4, respectively (Table 2 ). The trait coding TC1 was analyzed with a multiple threshold model and the trait coding TC2 and TC3 methods were analyzed with binary threshold models. A Bayesian single-trait threshold sire model and pedigree-derived relationship matrix of the sires were applied to these traits using the R-package MCMCglmm (Hadfield, 2010). Visual inspection of trace plots for all 3 trait coding approaches resulted in the use of 250,000 iterations, with a burn-in period of 100,000 and a thinning interval of 100 . The model was specified as follows:

$$
\lambda=\mathbf{X b}+\mathbf{Z s}+\mathbf{e},
$$

where $\mathbf{X}$ and $\mathbf{Z}$ are known incidence matrices, $\boldsymbol{\lambda}$ is a vector containing the sucking reflex liabilities of the calves, and vector $\mathbf{b}$ includes the fixed effects for herd, season (2 seasons), vigor, and size of the calves, with uniform priors for the effects in $\mathbf{b}$. The random effect of sire is denoted by $\mathbf{s}$, with a covariance structure $\boldsymbol{s} \mid \sigma_{s}^{2} \sim N\left(\mathbf{0}, \mathbf{A} \sigma_{s}^{2}\right)$ and a prior distribution of $\sigma_{s}^{2} \sim i n v-$ gamma $(0.01,0.01)$. This weak prior distribution is justified because the genetic variance of the trait is unknown. Matrix $\mathbf{A}$ is the pedigree-derived numerator relationship matrix, and $\sigma_{s}^{2}$ denotes the sire variance component. The residual variance e was set to 1 to ensure convergence. The heritability on the liability scale was defined as $\mathrm{h}^{2}=4 \sigma_{s}^{2} /\left(\sigma_{s}^{2}+\sigma_{e}^{2}\right)$, where $\sigma_{e}^{2}$ is the residual variance.

For illustrative purposes, for binary coding, the TC2 and TC3 sire effects were estimated by running the model a second time while keeping the variance components fixed at their estimated values. The posterior means of the sire effects on the liability scale were transformed to the observed scale as follows (Heringstad et al., 2004):

$$
p_{i}=\Phi\left(\mu+s_{i}\right),
$$

where $p_{i}$ is the probability of showing an insufficient sucking reflex of an infinite number of the expected trait value of animal $i, \Phi$ is the cumulative probability function of the standard normal distribution, $\mu$ is the probit function of the mean incidence (i.e., the mean liability), and $s_{i}$ is the sire effect estimated on the liability scale.

\section{Genome-Wide Association Studies}

The GWAS were conducted for the trait sucking reflex for trait coding TC1 by applying a single-marker and a Bayesian approach. Both models assumed normality of the data. Because TC2 and TC3 were binary, they were not considered in the GWAS. The phenotypes were adjusted for the fixed effects of herd, season (2 seasons), sex, size, and vigor of the calves. The sex of the calves was included in the GWAS models because the sex ratio of the genotype data was rather unbalanced. Pre-correction was performed using the phenotypic 
data set, which was larger than the genotypic data set, resulting in a more accurate pre-correction.

The single-marker GWAS was conducted using mixed linear model-based association analysis methods in GCTA (Yang et al., 2014). The model was as follows:

$$
\mathbf{y}=\mathbf{Z b}+\mathbf{g}+\mathbf{e},
$$

where $\mathbf{y}$ represents a vector of the response variable sucking reflex for each calf, vector $\mathbf{b}$ contains the fixed effect of the mean and the effect of the tested SNP, and $\mathbf{Z}$ is the design matrix. The SNP genotypes of the individuals were coded in $\mathbf{Z}$ as 1-allele copies (i.e., 0, 1, or 2 copies). The vector $\mathrm{g}$ is of dimension $\mathrm{n} \times 1$, containing a polygenic term, with $\mathbf{g} \sim N\left(\mathbf{0}, \mathbf{G} \sigma_{g}^{2}\right), \mathbf{G}$ being the genomic relationship matrix and $\sigma_{g}^{2}$ being the SNPbased variance. The $\mathbf{G}$ matrix was set up using all autosome SNP except the autosome harboring the SNP to be tested for association. This method accounts for double-fitting of the candidate SNP, which would result in a loss of power (Yang et al., 2014). The term $\mathbf{e}$ is the vector of random residual effect, with $\mathbf{e} \sim N\left(\mathbf{0}, \mathbf{I} \sigma_{e}^{2}\right)$, where $\mathbf{I}$ denotes the identity matrix and $\sigma_{e}^{2}$ denotes the residual variance.

For each SNP, a test statistic $P$-value was obtained. Correction for multiple testing was performed using Bonferroni correction. Two levels of significance were chosen (i.e., 5\% genome-wide after Bonferroni correction and $P \leq 5 \mathrm{E}-05$ ), without correction. The latter was also applied because Bonferroni correction is very conservative, especially for many dependent tests (as is the case in high-density SNP data). The Illumina SNP probe sequences of significant SNP were aligned to National Center for Biotechnology Information assembly Bos_taurus_UMD_3.1.1. to find positional candidate genes.

It is possible that the effect of a gene is only captured in part by a single marker due to imperfect LD and might be better explained jointly by the SNP surrounding the gene. Therefore, following Fernando and Garrick (2013), a multi-marker GWAS approach was applied, simultaneously fitting all SNP as random effects in the model. We used BayesC introduced by Verbyla et al. (2009) because this proved to be a suitable method for GWAS (Schmid and Bennewitz, 2017). The model is as follows:

$$
\mathbf{y}=\mathbf{1}_{\mathrm{n}} \boldsymbol{\mu}+\mathrm{X} \tilde{\mathbf{a}}+\mathbf{e},
$$

where $\mathbf{y}$ denotes the vector of the observations of the $n$ individuals, which were adjusted for the fixed effects described in model [1], $\mathbf{1}_{\mathrm{n}}$ is a vector of $\mathrm{n}$ ones, and $\boldsymbol{\mu}$ is the general mean. The vector $\tilde{\mathbf{a}}$ contains the random additive effects of $M$ SNP. $\mathbf{X}$ is an $\mathrm{n} \times \mathrm{M}$ matrix and contains the number of 1-allele copies at the SNP for each individual. The errors e are normally distributed. It was assumed that the distribution $F$ of the SNP effects was a mixture of $2 t$-distributions that differed only by a scaling factor, $\varepsilon$. Conditionally, on a Bernoulli-distributed indicator variable, $\gamma_{j}$, it follows that $\tilde{a}_{j} \mid \gamma_{j} \sim \gamma_{j} F+\left(1-\gamma_{j}\right) \varepsilon F$. Thus, if $\gamma_{j}=1$, the marker effect comes from the distribution with the larger variance. The prior probability of a marker being important (i.e., $\gamma_{j}=1$ ) was $p L D$. The distribution of the additive effects was a $t$-distribution. We chose $\varepsilon=0.01, p L D=$ 0.05 , and $3.5 \mathrm{df}$ for the $t$-distribution. A single Markov chain Monte Carlo chain was run for 150,000 cycles, discarding the first 50,000 as burn-in. The R-package BayesDsamples (Wellmann and Bennewitz, 2012) was applied. The posterior SNP effect estimates were used to calculate the genomic breeding values for sliding windows with a size of $1 \mathrm{cM}\left(\mathrm{EBV}_{\mathrm{W}}\right)$, assuming that 1 $\mathrm{Mb}$ corresponds roughly to $1 \mathrm{cM}$. The deviation of the variance of the $\mathrm{EBV}_{\mathrm{W}}$ from the expected variance was used to pinpoint trait-associated chromosomal regions (Bennewitz et al., 2017). Adjacent chromosomal regions showing a window variance deviation $\geq 2 \mathrm{E}-05$ were presumed to contain a region of putative QTL. The detected QTL regions were compared with the results from single-marker GWAS. Genes annotated in QTL regions were listed as positional candidate genes for sucking reflex.

\section{Post-GWAS Analysis}

Genes in regions of $1 \mathrm{Mb}$ upstream and downstream of significant SNP positions from GWAS results were identified and further analyzed. Over- or underrepresentation of Gene Ontologies (GO) for biological processes was statistically tested applying Fisher's exact test in the PANTHER database (protein analysis through evolutionary relationships; Thomas et al., 2006; Mi et al., 2013, 2017). False discovery rates were calculated to correct for multiple testing. Furthermore, gene enrichment and pathway analysis was conducted using functional annotation clustering and functional annotation chart tools implemented in the Database for Annotation, Visualization and Integrated Discovery (DAVID; Huang et al., 2009a,b). For functional cluster annotations, clusters with a kappa similarity threshold $\leq 0.70$ and an enrichment score $\leq 1.3(P \geq 0.05)$ were discarded. Pathway analysis was done with Kyoto Encyclopedia of Genes and Genomes (Kanehisa and Goto, 2000) and Reactome (Fabregat et al., 2018) pathways using DAVID functional annotation chart tool. 


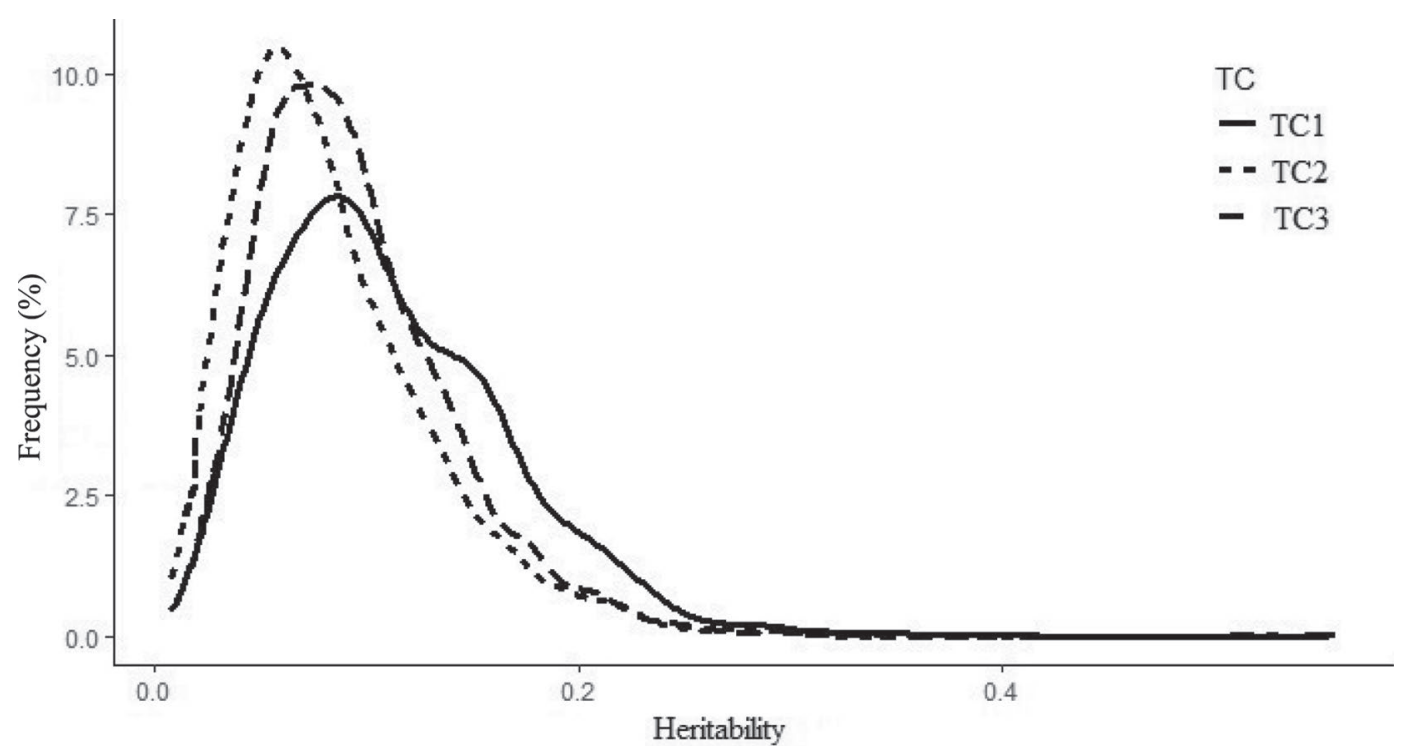

Figure 1. Posterior distributions of heritability for the different sucking reflex trait codings (TC1, TC2, and TC3) with heritability estimates on the $\mathrm{x}$-axis.

\section{RESULTS}

The distribution of the trait sucking reflex is shown in Table 1. Approximately 9.5\% of the newborn calves showed no sucking reflex at all, and $14 \%$ had only a weak sucking reflex, illustrating that this is a serious problem in the Brown Swiss dairy population. Colostrum intake by means of esophageal tubes had to be done for $9 \%$ of the young stock. We found no significant differences regarding the sex of the calves. The effects of size and vigor were highly significant $(P<0.001)$. Larger calves and calves with a stronger vigor showed fewer sucking problems. Both the farm effect and the season effect (tested for the summer and winter seasons) were highly significant $(P<0.001)$. Fewer sucking problems occurred during the summer season.

The heritability estimates obtained from the threshold models are shown in Table 2. The TC1 resulted in a slightly higher heritability for the trait than the values of TC2 and TC3. The trace plots, as well as the shape of the heritability posterior distributions (Figure 1), revealed the convergence of the model. The heritability 95\% support intervals were wide (Table 2), and thus, the estimates must be treated with some caution. The plot of the posterior mean sire effects on a probability scale for TC2 and TC3 is shown in Figure 2.

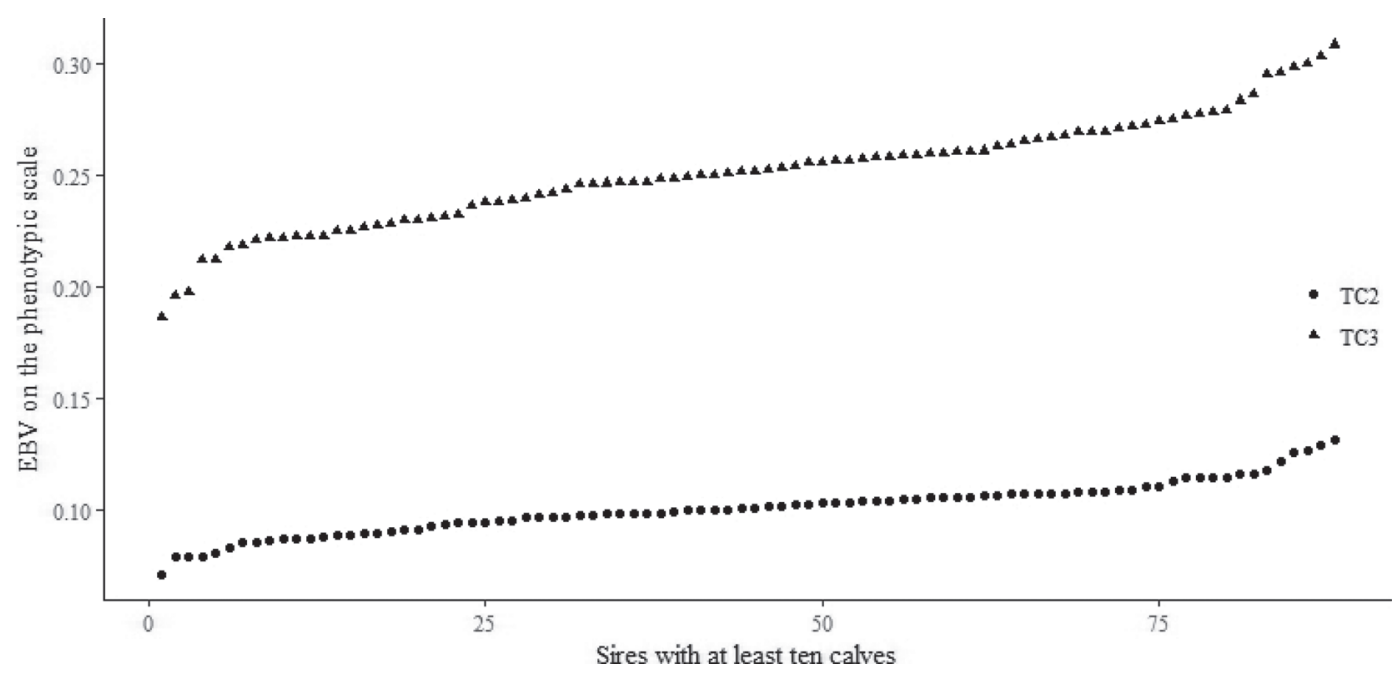

Figure 2. Plot of the average posterior sire mean on the probability scale for sucking reflex trait codings TC2 and TC3. 
The GWAS results for TC 1 are shown in Figure 3. No SNP reached the genome-wide significance level in the single-marker analysis. We found a total of 34 significant SNP on BTA 7, 9, 11, 14, 15, and 25 for TC 1. A list of SNP names and positions for SNP with $P \leq 5 \mathrm{E}-05$ is given in Supplemental Table S1 (https: //doi.org/10.3168/jds.2019-16487). The Bayesian multi-marker GWAS produced the strongest signals on BTA 7, 15, and 21. Table 3 shows a comparison between SNP regions and positions with peak results in multi-marker and single-marker GWAS and positional candidate genes located in these regions. A total of 277 genes were identified in the $1 \mathrm{Mb}$ up- and downstream regions of detected SNP (Table 4). The DAVID mapped all 277 genes in post-GWAS analyses, whereas PANTHER mapped only 173 genes to PANTHER GO terms with biological processes. Test of over- or underrepresentation of GO terms in PANTHER revealed 39 statistically significant $(P \leq 0.01)$ GO terms for biological processes. Supplemental Table S2 (https:// doi.org/10.3168/jds.2019-16487) shows the results for the test of over- or underrepresentation of GO terms in PANTHER. Highest significance was found for the overrepresentation for central nervous system development (GO: 0007417). Moreover, we found the overrepresentation of GO terms with functions in the adapted immune system or in the regulation of lipid metabolic process. Response to stimulus (GO: 0050896) was the only biological process significantly underrepresented.

Functional annotation clustering with DAVID revealed a total of 6 clusters (Supplemental Tables S3.1 to S3.6; https://doi.org/10.3168/jds.2019-16487), with functions in humoral immunity (cluster 1) and cellular immunity (cluster 5), development of the central nervous system and skeletal system (cluster 3), or lipid metabolic processes (cluster 2 and 4). Pathway analysis also revealed significant pathways for the immune system, endocrine system, and transport of small molecules (Supplemental Table S4; https://doi.org/10 .3168/jds.2019-16487).

\section{DISCUSSION}

In this study, we clearly showed that an insufficient sucking reflex is a serious problem in the dairy Brown Swiss cattle breed. The incidence is similar to that reported by Maltecca et al. (2007). The trait showed a low heritability up to $11 \%$, which is slightly below the estimates from Maltecca et al. (2007); however, the trait definition also differed. Brown Swiss farmers in Germany want the sucking reflex to be included as a new trait in the selection index (Just et al., 2018). The heritability estimates suggest that the selection for this trait would indeed result in a selection response. The plots of the sire posterior mean on the probability scale (Figure 2) illustrate the existence of some useable estimated breeding value variation for the improvement of the trait. Additionally, the GWAS results clearly show that the sucking reflex of calves is a complex trait with many genes involved. Thus, if the trait sucking reflex would be included in the selection index, routine data collection and routine breeding value estimation are indispensable, and for the estimation of reliable breeding values, large progeny testing groups or a large reference population would be required in a genomic selection program. Because we found that maternal effects seemed to have an effect on the calves' sucking reflex as well (ongoing research project, data not shown), it is recommended to also estimate the breeding values for direct and maternal effects.

The trace element selenium plays a major role in several pathways of signal transduction and nerve cell development and is attributed to an important antioxidant effect in the protection of cells from oxidative stress and damage (McKenzie et al., 2002; Konvičná and Kováć, 2015). Because the transition period of dairy cows is often a stage with a higher risk of oxidative stress, an insufficient selenium supply or defective absorption capacity of the dam during this risky period might also lead to a decreased function of the calf s sucking reflex. The evaluation of selenium blood levels in calves and dams would be interesting for further studies on the impact of maternal effects on the calves' sucking reflex.

Different trait coding resulted in slightly different results. It seems that $\mathrm{TC} 1$ and $\mathrm{TC} 3$ were beneficial because the heritability estimates were slightly larger. Treating "no" and "weak" sucking reflex as different classes is advisable because the problems associated with insufficient sucking are mostly due to the complete absence of the reflex, measured as trait category "no." The poorer results in TC2 might be due to the unbalanced records when comparing the calves with no sucking reflex at all with the combination of the remaining categories.

Compared with the studies of Vasseur et al. (2009), we confirmed that the size and vigor of a calf have the strongest influence on its ability to develop a healthy sucking reflex. Homerosky et al. (2017) measured the sucking reflex in beef calves and proved that vigor and calving ease had a relationship to the calf's sucking reflex. Calving ease had no significant effect on the sucking reflex in our findings; instead, we included the calf's size and vigor as explanatory variables in the models. This might be suboptimal because these are heritable traits themselves, and thus, correcting for them would lower the genetic variance. However, we were interested in the direct genetic effects causing 

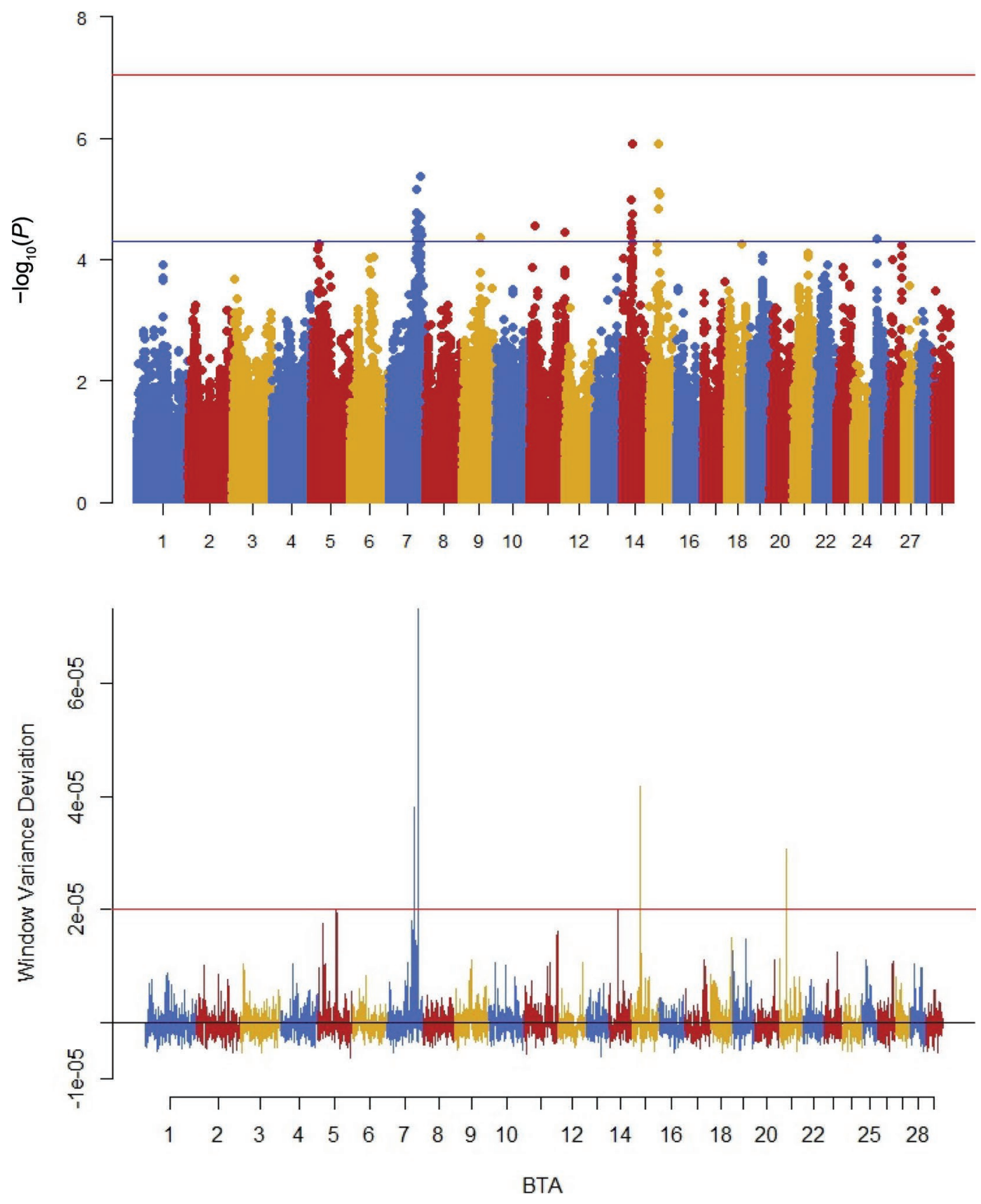

Figure 3. Genome-wide association study (GWAS) results for the trait sucking reflex TC1. Top: Manhattan plot of single-marker GWAS (blue solid line is the threshold for nominal $P \leq 5 \mathrm{E}-05$, red solid line is the threshold for genome-wide significance). Bottom: Window variance deviations obtained from Bayesian multi-marker GWAS. Red solid line is drawn at 2E-05.

improper sucking reflex and did not consider indirect effects, mediated via small and weak calves. For the future estimation of breeding values, it is more convenient to include the gestation length instead of calf size and vigor as fixed effects in the analyses. Indeed, the inclusion of gestation length showed no significance when the effects of size and vigor of the calves were left in the model; however, gestation length was significant when size and vigor were excluded. Including gestation lengths in the model also has advantages from a practical point of view because gestation lengths are usually available from computing centers, whereas the size and vigor of the calves must be recorded by the farmers as additional traits. We tested the substitution of size and 
Table 3. Quantitative trait loci found in multi-marker GWAS, positional candidate genes, and SNP in these regions with $P \leq 5 \mathrm{E}-05$ from singlemarker genome-wide association studies (GWAS)

\begin{tabular}{|c|c|c|c|c|c|c|}
\hline \multicolumn{5}{|c|}{ Multi-marker GWAS } & \multicolumn{2}{|c|}{ Single-marker GWAS } \\
\hline $\begin{array}{l}\text { QTL } \\
\text { no. }\end{array}$ & BTA & Start & End & Positional candidate genes & $\begin{array}{c}\text { RefSeq } \\
\text { number }\end{array}$ & $\begin{array}{l}\text { Position } \\
\text { (bp) }\end{array}$ \\
\hline 1 & 7 & $86,593,875$ & $86,633,971$ & EDIL3 & $\begin{array}{r}137,246,368 \\
136,980,239 \\
42,301,774\end{array}$ & $\begin{array}{l}86,575,688 \\
86,582,012 \\
86,593,875\end{array}$ \\
\hline 2 & 7 & $98,454,085$ & $98,985,065$ & CAST, ERAP1, ERAP2, LNPEP, LIX1 & $\begin{array}{l}132,707,186 \\
110,540,286 \\
135,811,099\end{array}$ & $\begin{array}{l}98,448,831 \\
98,449,505 \\
98,474,995\end{array}$ \\
\hline 3 & 15 & $28,496,787$ & $28,947,625$ & CEP164, DSCAML1, FXYD6 & $\begin{array}{r}41,758,723 \\
41,758,721 \\
41,758,719 \\
41,758,714\end{array}$ & $\begin{array}{l}28,495,074 \\
28,495,571 \\
28,497,908 \\
28,501,479\end{array}$ \\
\hline 4 & 21 & $20,282,153$ & $21,041,394$ & $\begin{array}{l}\text { LOC101906218, LOC100298937, LOC100336807, } \\
\text { LOC515149, LOC101906604, ACAN, HAPLN3, } \\
\text { MFGE8, ABHD2 }\end{array}$ & - & - \\
\hline
\end{tabular}

vigor by gestation length and gestation length squared in model [1] and estimated almost the same heritabilities (not shown elsewhere).

Because of the low heritability and the large farm effects, it becomes obvious that primarily nongenetic effects cause the insufficient sucking reflex. The farm effect can be divided into farm person effects and farm managing effects. The former consists of the individual judgment of the sucking reflex and the treatment of newborn calves by the stockperson. The latter might include factors such as the housing and feeding regimen of the cows. More research is needed to unravel the farm effects in detail and to identify the factors that caused the different farm incidences.

The results of multi-marker and single-marker GWAS revealed a quantitative genetic background of the trait. We detected some genomic regions containing putative QTL. A comparison between both GWAS models revealed some similar regions with positional candidate genes (Table 3). The genetic background of a nonfunctional sucking reflex might be explained using

Table 4. Positions of $1-\mathrm{Mb}$ regions up- and downstream from significant SNP from both genome-wide association study models and total number of genes located in these regions

\begin{tabular}{lcc}
\hline BTA & Region $(\mathrm{bp})$ & No. of genes \\
\hline 7 & $85,575,688-87,593,875$ & 11 \\
7 & $97,448,831-99,474,995$ & 25 \\
9 & $56,747,539-58,747,539$ & 7 \\
11 & $15,152,298-17,152,298$ & 16 \\
11 & $105,029,236-107,029,236$ & 84 \\
14 & $28,594,745-34,640,136$ & 47 \\
15 & $27,495,074-29,501,479$ & 48 \\
15 & $30,731,747-32,731,747$ & 24 \\
21 & $20,282,153-21,041,394$ & 9 \\
25 & $10,841,482-12,841,482$ & 6 \\
Sum & & 277 \\
\hline
\end{tabular}

the results of the post-GWAS analysis. The incorrect transduction of sensor stimuli or conduction of nervous impulses could prevent a calf from developing a functional sucking reflex. Indeed, the highest significance was tested for GO term of central nervous system development (Supplemental Table S2; https://doi.org/10 .3168/jds.2019-16487). We detected regions inheriting genes with functions in neurogenesis, brain development, and signal transduction in nerve cells, such as DSCAML1, HAPLN1, HAPLN3, ACAN, and VCAN. Clusters 2 and 3 (Supplemental Tables S3.2 and S3.3; https://doi.org/10.3168/jds.2019-16487) inherit GO terms associated with the development of the central nervous system, endocrine system, and lipid metabolism. Results of pathway analysis also detected genes with functions in lipid metabolism or endocrine system (Supplemental Table S4; https://doi.org/10.3168/jds .2019-16487). It is known that the lipid metabolism in human brain is critical during neurogenesis and for the maintenance of efficient signal transmission by means of myelinated axons. Furthermore, neurodegenerative diseases in humans are often associated with defective lipid metabolism (Bales, 2010; Shamim et al., 2018). A more comprehensive analysis of further reflexes would be helpful to confirm an overall malfunction of the central nervous system.

A further explanation would be a calf s erroneous or missing perception of hunger, leading to an insufficient sucking reflex. This suggestion is supported by a candidate gene [i.e., corticotropin-releasing hormone $(C R H)$ on BTA 14]. The $C R H$ is not only involved in stress responses and locomotory exploration behaviors but also affects food intake and physiological measures associated with food intake (Glowa et al., 1992; Arborelius et al., 1999; Bernier, 2006). A further assumption of the genetic background for an erroneous sucking 
reflex was the defective olfactory perception of nonsucking calves. Earlier studies in pigs and mice have already suggested that the olfactory system is essential for nipple-searching behavior and inducing sucking reflex (Morrow-Tesch and McGlone, 1990; Hongo et al., 2000). Nevertheless, this assumption could not be confirmed in our study.

\section{CONCLUSIONS}

The results of this study showed that an insufficient sucking reflex is a problem in the Brown Swiss population. Heritability was estimated up to $11 \%$, and the GWAS results showed that this is a typical quantitative trait. The heritability estimates suggest that breeding for an improved sucking reflex is possible but requires large data sets for the estimation of reliable breeding values (either large progeny testing groups or a large reference genome in a genomic selection program). The application of a 4-stage ordinal trait coding is suggested for routine data recording. Future research is needed to unravel the large nongenetic and farm effects as well as to analyze putative maternal effects.

\section{ACKNOWLEDGMENTS}

The authors thank the staff members of the Rinderunion Baden-Wuerttemberg e.V. (Herbertingen, Germany), the participating farmers (located in the state Baden-Wuerttemberg, Germany), and the Foerderverein Biooekonomieforschung e. V. (Bonn, Germany) for excellent cooperation. The study was financially supported by Landwirtschaftliche Rentenbank (Frankfurt a. M., Germany).

\section{REFERENCES}

Arborelius, L., M. J. Owens, P. M. Plotsky, and C. B. Nemeroff. 1999. The role of corticotropin-releasing factor in depression and anxiety disorders. J. Endocrinol. 160:1-12.

Bales, K. R. 2010. Brain lipid metabolism, apolipoprotein E and the pathophysiology of Alzheimer's disease. Neuropharmacology 59:295-302. https://doi.org/10.1016/j.neuropharm.2010.01.005.

Beam, A. L., J. E. Lombard, C. A. Kopral, L. P. Garber, A. L. Winter, J. A. Hicks, and J. L. Schlater. 2009. Prevalence of failure of passive transfer of immunity in newborn heifer calves and associated management practices on US dairy operations. J. Dairy Sci. 92:3973-3980. https://doi.org/10.3168/jds.2009-2225.

Bennewitz, J., C. Edel, R. Fries, T. H. E. Meuwissen, and R. Wellmann. 2017. Application of a Bayesian dominance model improves power in quantitative trait genome-wide association analysis. Genet. Sel. Evol. 49:7. https://doi.org/10.1186/s12711-017-0284-7.

Bernier, N. J. 2006. The corticotropin-releasing factor system as a mediator of the appetite-suppressing effects of stress in fish. Gen. Comp. Endocrinol. 146:45-55.

Bostedt, H., and P. Schramel. 1990. The importance of selenium in the prenatal and postnatal development of calves and lambs. Biol. Trace Elem. Res. 24:163-171. https://doi.org/10.1007/ BF02917204.
Browning, B. L., and S. R. Browning. 2009. A unified approach to genotype imputation and haplotype-phase inference for large data sets of trios and unrelated individuals. Am. J. Hum. Genet. 84:210-223. https://doi.org/10.1016/j.ajhg.2009.01.005.

Butler, D. 2009. asreml: asreml() fits the linear mixed model. R package version 3.0. www.vsni.co.uk.

Fabregat, A., S. Jupe, L. Matthews, K. Sidiropoulos, M. Gillespie, P. Garapati, R. Haw, B. Jassal, F. Korninger, B. May, M. Milacic, C. D. Roca, K. Rothfels, C. Sevilla, V. Shamovsky, S. Shorser, T. Varusai, G. Viteri, J. Weiser, G. Wu, L. Stein, H. Hermjakob, and P. D'Eustachio. 2018. The Reactome Pathway Knowledgebase. Nucleic Acids Res. 46(D1):D649-D655. https://doi.org/10.1093/ nar/gkx1132.

Fernando, R. L., and D. Garrick. 2013. Bayesian methods applied to GWAS. Methods Mol. Biol. 1019:237-274. https://doi.org/10 .1007/978-1-62703-447-0_10.

Glowa, J. R., J. E. Barrett, J. Russell, and P. W. Gold. 1992. Effects of corticotropin releasing hormone on appetitive behaviors. Peptides 13:609-621. https://doi.org/10.1016/0196-9781(92)90097-M.

Gomez, D., and M. Chamorro. 2017. The importance of colostrum for dairy calves. Rev. Colomb. Cienc. Pecu. 30:241-244.

Hadfield, J. D. 2010. MCMC methods for multi-response generalized linear mixed models: The MCMCglmm R package. J. Stat. Softw. $33: 1-22$.

Hadorn, U., H. Hammon, R. M. Bruckmaier, and J. W. Blum. 1997. Delaying colostrum intake by one day has important effects delaying colostrum intake by one day has important effects on metabolic traits and on gastrointestinal and metabolic hormones in neonatal calves. J. Nutr. 127:2011-2023.

Hefnawy, A. E. G., and J. L. Tórtora-Pérez. 2010. The importance of selenium and the effects of its deficiency in animal health. Small Rumin. Res. 89:185-192. https://doi.org/10.1016/j.smallrumres .2009 .12 .042 .

Heringstad, B., Y. M. Chang, D. Gianola, and G. Klemetsdal. 2004. Multivariate threshold model analysis of clinical mastitis in multiparous Norwegian dairy cattle. J. Dairy Sci. 87:3038-3046. https: //doi.org/10.3168/jds.S0022-0302(04)73436-0.

Homerosky, E. R., E. Timsit, E. A. Pajor, J. P. Kastelic, and M. C. Windeyer. 2017. Predictors and impacts of colostrum consumption by $4 \mathrm{~h}$ after birth in newborn beef calves. Vet. J. 228:1-6. https:// doi.org/10.1016/j.tvjl.2017.09.003.

Hongo, T., A. Hakuba, K. Shiota, and I. Naruse. 2000. Suckling dysfunction caused by defects in the olfactory system in genetic arhinencephaly mice. Biol. Neonate 78:293-299. https://doi.org/ $10.1159 / 000014282$.

Howie, B., C. Fuchsberger, M. Stephens, J. Marchini, and G. R. Abecasis. 2012. Fast and accurate genotype imputation in genomewide association studies through pre-phasing. Nat. Genet. 44:955959. https://doi.org/10.1038/ng.2354.

Huang, W., B. T. Sherman, and R. A. Lempicki. 2009a. Bioinformatics enrichment tools: Paths toward the comprehensive functional analysis of large gene lists. Nucleic Acids Res. 37:1-13. https://doi .org/10.1093/nar/gkn923.

Huang, W., B. T. Sherman, and R. A. Lempicki. 2009b. Systematic and integrative analysis of large gene lists using DAVID bioinformatics resources. Nat. Protoc. 4:44-57. https://doi.org/10.1038/ nprot.2008.211.

Just, A., R. Wellmann, and J. Bennewitz. 2018. Estimation of relative economic weights and the marginal willingness to pay for breeding traits of Brown Swiss cattle using discrete choice experiments. J. Dairy Sci. 101:5207-5213. https://doi.org/10.3168/jds.2017-14012.

Kanehisa, M., and S. Goto. 2000. KEGG: Kyoto encyclopedia of genes and genomes. Nucleic Acids Res. 28:27-30.

Konvičná, J., and G. Kováč. 2015. Oxidative stress and role of selenium during peripaturient period in dairy cows. Folia Vet. 59:44-52.

Maltecca, C., A. Rossoni, C. Nicoletti, E. Santus, K. A. Weigel, and A. Bagnato. 2007. Estimation of genetic parameters for perinatal sucking behavior of Italian Brown Swiss calves. J. Dairy Sci. 90:4814-4820. https://doi.org/10.3168/jds.2007-0183.

McKenzie, R. C., J. R. Arthur, and G. J. Beckett. 2002. Selenium and the regulation of cell signaling, growth, and survival: Molecular 
and mechanistic aspects. Antioxid. Redox Signal. 4:339-351. https: //doi.org/10.1089/152308602753666398.

Mi, H., X. Huang, A. Muruganujan, H. Tang, C. Mills, D. Kang, and P. D. Thomas. 2017. PANTHER version 11: Expanded annotation data from Gene Ontology and Reactome pathways, and data analysis tool enhancements. Nucleic Acids Res. 45(D1):D183-D189. https://doi.org/10.1093/nar/gkw1138.

Mi, H., A. Muruganujan, J. T. Casagrande, and P. D. Thomas. 2013. Large-scale gene function analysis with the PANTHER classification system. Nat. Protoc. 8:1551-1566. https://doi.org/10.1038/ nprot.2013.092.

Morrow-Tesch, J., and J. J. McGlone. 1990. Sensory systems and nipple attachment behavior in neonatal pigs. Physiol. Behav. 47:1-4. https://doi.org/10.1016/0031-9384(90)90034-2.

Purcell, S., B. Neale, K. Todd-Brown, L. Thomas, M. A. R. Ferreira, D. Bender, J. Maller, P. Sklar, P. I. W. de Bakker, M. J. Daly, and P. C. Sham. 2007. PLINK: A tool set for whole-genome association and population-based linkage analyses. Am. J. Hum. Genet. 81:559-575. https://doi.org/10.1086/519795.

Reed, J. J., M. A. Ward, K. A. Vonnahme, T. L. Neville, S. L. Julius, P. P. Borowicz, J. B. Taylor, D. A. Redmer, A. T. Grazul-Bilska, L. P. Reynolds, and J. S. Caton. 2007. Effects of selenium supply and dietary restriction on maternal and fetal body weight, visceral organ mass and cellularity estimates, and jejunal vascularity in pregnant ewe lambs. J. Anim. Sci. 85:2721-2733. https://doi.org/ $10.2527 /$ jas.2006-785.

Schmid, M., and J. Bennewitz. 2017. Invited review: Genome-wide association analysis for quantitative traits in livestock-A selective review of statistical models and experimental designs. Arch. Tierzucht 60:335-346. https://doi.org/10.5194/aab-60-335-2017.

Schwarzenbacher, H., J. Burgstaller, F. R. Seefried, C. Wurmser, M. Hilbe, S. Jung, C. Fuerst, N. Dinhopl, H. Weissenboeck, B. FuerstWaltl, M. Dolezal, R. Winkler, O. Grueter, U. Bleul, T. Wittek, R. Fries, and H. Pausch. 2016. A missense mutation in TUBD1 is associated with high juvenile mortality in Braunvieh and Fleckvieh cattle. BMC Genomics 17:400.

Shamim, A., T. Mahmood, F. Ahsan, A. Kumar, and P. Bagga. 2018. Lipids: An insight into the neurodegenerative disorders. Clin. Nutr. Exp. 20:1-19. https://doi.org/10.1016/j.yclnex.2018.05.001. Thomas, P. D., A. Kejariwal, N. Guo, H. Mi, M. J. Campbell, A Muruganujan, and B. Lazareva-Ulitsky. 2006. Applications for protein sequence-function evolution data: MRNA/protein expression analysis and coding SNP scoring tools. Nucleic Acids Research 34(Web Server):W645-W650. https://doi.org/10.1093/nar/ gkl229.

Vasseur, E., J. Rushen, and A. M. de Passillé. 2009. Does a calf's motivation to ingest colostrum depend on time since birth, calf vigor, or provision of heat? J. Dairy Sci. 92:3915-3921. https://doi.org/ $10.3168 /$ jds.2008-1823.

Verbyla, K. L., B. J. Hayes, P. J. Bowman, and M. E. Goddard. 2009. Accuracy of genomic selection using stochastic search variable selection in Australian Holstein Friesian dairy cattle. Genet. Res. (Camb.) 91:307-311. https://doi.org/10.1017/S0016672309990243.

Weaver, D. M., J. W. Tyler, D. C. VanMetre, D. E. Hostetler, and G. M. Barrington. 2000. Passive transfer of colostral immunoglobulins in calves. J. Vet. Intern. Med. 14:569-577.

Wellmann, R., and J. Bennewitz. 2012. Bayesian models with dominance effects for genomic evaluation of quantitative traits. Genet. Res. 94:21-37.

Windeyer, M. C., K. E. Leslie, S. M. Godden, D. C. Hodgins, K. D. Lissemore, and S. J. LeBlanc. 2014. Factors associated with morbidity, mortality, and growth of dairy heifer calves up to 3 months of age. Prev. Vet. Med. 113:231-240. https://doi.org/10.1016/j .prevetmed.2013.10.019.

Yang, J., N. A. Zaitlen, M. E. Goddard, P. M. Visscher, and A. L. Price. 2014. Advantages and pitfalls in the application of mixedmodel association methods. Nat. Genet. 46:100-106. https://doi .org/10.1038/ng.2876. 